\title{
(C) OPEN ACCESS \\ Evaluation of vascular changes in intermediate uveitis and retinal vasculitis using swept-source wide-field optical coherence tomography angiography
}

\author{
Meng Tian, ${ }^{1}$ Christoph Tappeiner, ${ }^{1}$ Martin S Zinkernagel, ${ }^{1}$ Wolfgang Huf, ${ }^{2,3}$ \\ Sebastian Wolf, ${ }_{1}^{1,4}$ Marion R Munk ${ }^{1,4}$
}

\begin{abstract}
- Additional material is published online only. To view please visit the journal online (http://dx.doi.org/10.1136/ bjophthalmol-2018-313078).

${ }^{1}$ Department of Ophthalmology, Inselspital, Bern University Hospital, University of Bern, Bern, Switzerland

${ }^{2}$ Karl Landsteiner Institute for Clinical Risk Management,

Vienna, Austria

${ }^{3}$ Department of Laboratory Medicine, Hietzing Hospital, Vienna, Austria

${ }^{4}$ Bern Photographic Reading Center, Inselspital, Bern University Hospital, University of Bern, Bern, Switzerland
\end{abstract}

\section{Correspondence to}

Dr Marion R Munk, Department of Ophthalmology, Inselspital, Bern University Hospital, Switzerland; marion_munk@ hotmail.com

Received 14 August 2018 Revised 19 October 2018 Accepted 29 October 2018 Published Online First 11 December 2018 University of Bern, Bern 3010,

\begin{abstract}
Purpose To evaluate vascular changes in patients with intermediate uveitis with or without retinal vasculitis using swept-source wide-field optical coherence tomography angiography (OCTA).

Methods This is a prospective cross-sectional study. Consecutive patients with intermediate uveitis were evaluated using wide-field OCTA. Wide-field OCTA and en-face OCT images were analysed for the presence of capillary non-perfusion and reduced perfusion, disruption of ellipsoid zone, and abnormalities on en-face wide-field retinal thickness maps, respectively, and compared with fluorescein angiography (FA) findings in a subcohort. Results 164 eyes of 88 patients with intermediate uveitis were included. Areas of capillary non-perfusion and reduced perfusion were more frequently observed in the choroidal OCTA slab (33.3\% and $49.4 \%)$, choriocapillaris (CC; $31.4 \%$ and $48 \%$ ) and deep capillary plexus (DCP; $9.6 \%$ and $34.6 \%$ ) than in the superficial capillary plexus (SCP; $5 \%$ and $26.3 \%$ ), respectively. Intermediate uveitis with vasculitis presented more frequently with non-perfusion and hypoperfusion in the DCP ( $p=0.003$ and $p=0.05$, respectively) and SCP ( $p=0.007$ and $p=0.005$, respectively) than intermediate uveitis without vasculitis. Peripheral capillary leakage on FA correlated with the presence of perivascular, macular and generalised thickening on en-face widefield thickness maps ( $p=0.007)$. Ischaemia on FA was significantly associated with non-perfusion on widefield OCTA in SCP and DCP ( $p=0.019$ and $p=0.027$, respectively).

Conclusion Changes in the choroid, CC and DCP are more frequently found than in the SCP on wide-field OCTA in intermediate uveitis. While wide-field OCTA is a reliable tool to detect capillary non-perfusion in intermediate uveitis, it was not helpful in determining disease activity.

Trial registration number NCT02811536.
\end{abstract}

\section{INTRODUCTION}

(c) Author(s) (or their employer(s)) 2019. Re-use permitted under CC BY-NC. No commercial re-use. See rights and permissions. Published by BMJ.

To cite: Tian M, Tappeiner $C$, Zinkernagel MS, et al. Br J Ophthalmol

2019:103:1289-1295.
Intermediate uveitis (IU) is a type of uveitis mainly localised to the vitreous and the ciliary body that lacks chorioretinal inflammation. ${ }^{1}$ Concomitant retinal vasculitis is often found in this disease, involving commonly the peripheral retinal veins.

Fluorescein angiography (FA) is sensitive in the diagnosis of retinal vasculitis and also an important diagnostic tool to detect macular oedema, optic disc oedema and other complications. ${ }^{2}$ However, FA is invasive and has a substantially limited depth resolution; thus, the middle and deeper capillary plexus are difficult to visualise. ${ }^{3}$ Moreover, leakage of dye can limit our ability to evaluate adjacent capillary perfusion. ${ }^{4}$ Indocyanine green angiography (ICG) allows the assessment of uveitis with choroidal involvement. However, the exact localisation of the inflammation within the choroid using ICG may be challenging. ${ }^{5}$

Optical coherence tomography angiography (OCTA) is a new, non-invasive imaging method that provides volumetric data of retinal and choroidal layers. ${ }^{6}$ OCTA has already shown its advantage in evaluating foveal microvascular changes in retinal vascular diseases as it is able to visualise vascular flow in the deep capillary plexus (DCP) as well as in the choriocapillaris (CC) and the choroid. ${ }^{6}$ This may be of importance as previous studies could illustrate that the deeper vessels rather than the superficial vascular plexus are more affected and impaired in retinal vascular diseases such as diabetic retinopathy. ${ }^{7}$ Furthermore vascular changes may be more easily detected using OCTA compared with other imaging modalities. ${ }^{8}$ Moreover, OCTA can provide separate evaluation of abnormalities in the retinal and choroidal circulations. ${ }^{9}$

The wide-field OCTA improves the narrow field of view of traditional OCTA. Previous studies $^{10} 11$ have shown that wide-field OCTA successfully detected vascular abnormalities seen on FA in retinal diseases such as diabetic retinopathy and polypoidal choroidal vasculopathy. But inherently OCTA cannot detect vascular staining or leakage, and its role therefore is somewhat different in uveitis than in other vascular disorders dominated by structural changes such as capillary loss and others.

The aim of our study is therefore to identify and evaluate vascular changes in patients with IU with or without retinal vasculitis using wide-field OCTA and compare findings with wide-field FA in a subcohort.

\section{METHODS \\ Study population}

This was a prospective cross-sectional study. Consecutive patients presenting with IU with or without retinal vasculitis at the Department of Ophthalmology of Inselspital, University of Bern, between October 2016 and October 2017 were enrolled in this study. 
Subjects were eligible if the following criteria were met: adult patients diagnosed with non-infectious IU with or without retinal vasculitis. The diagnosis of IU fulfilled the criteria of the Standardization in Uveitis Nomenclature $(\mathrm{SUN})^{12}$ and the disease activity was based on the National Eye Institute (NEI) system. ${ }^{13}$ The diagnosis of retinal vasculitis was based on the ophthalmoscopic criteria of a history or presence of intraocular inflammation, perivascular exudates, intraretinal haemorrhage or cotton-wool spots on ophthalmoscopy and evidence of vascular occlusion or profound leakage on fluorescein angiogram (FA). ${ }^{14} 15$ Concomitant peripheral vascular sheathing as regularly seen in IU was not deemed to be retinal vasculitis. Investigational work-up included chest X-ray, QuantiFERON-TB Gold in tube test, serological testing for syphilis and Lyme disease, and immunology tests including HLA-B51, antinuclear antibodies, ACE, lysozyme, interleukin-2 receptor and antineutrophil cytoplasmic antibodies. Furthermore, an MRI of the brain was performed to screen for cerebral vasculitis and demyelinating lesions.

Subjects with any ocular condition that would interfere with good-quality image acquisition were excluded. Patients with history of ocular trauma or other retinal diseases, such as diabetic retinopathy, hypertensive retinopathy, central serous chorioretinopathy and macular degeneration, and optic nerve diseases such as glaucoma or optic neuropathy, were also excluded.

\section{Acquisition of OCTA image}

All patients underwent ophthalmic examination including Snellen best-corrected visual acuity, slit-lamp examination, intraocular pressure and dilated fundus examination. Wide-field FA was performed with Heidelberg Spectralis HRA+OCT (Heidelberg Engineering, Heidelberg, Germany) using the $102^{\circ}$ lens. At 5 min peripheral sweeps of all quadrants were acquired. If FA was not performed on the same day, the most recent FA images were compared with OCTA images; in this case, the FAs were only included for comparison, if there was no reported change in disease activity or therapy and performance of FA was not more than 28 days apart from OCTA acquisition. ICG imaging, which was typically performed for initial work-up, was also reviewed to rule out posterior involvement.

All patients underwent imaging with the swept-source (SS)OCT (PLEX Elite 9000; Carl Zeiss Meditec, Dublin, California). Patients were imaged using wide-field montage OCTA scan consisting of five $12 \times 12 \mathrm{~mm}$ scans, covering about $80^{\circ}-90^{\circ}$ of the posterior pole (one centred on the fovea, the others centred on the temporal-superior, temporal-inferior, nasal-superior and nasal-inferior quadrants; figure 1). The individual $12 \times 12 \mathrm{~mm}$ scans were reviewed for the presence of alignment errors and artefacts due to floaters and manually corrected if present. After quality check the $12 \times 12$ images were montaged using the automatic montage export function available on the device.

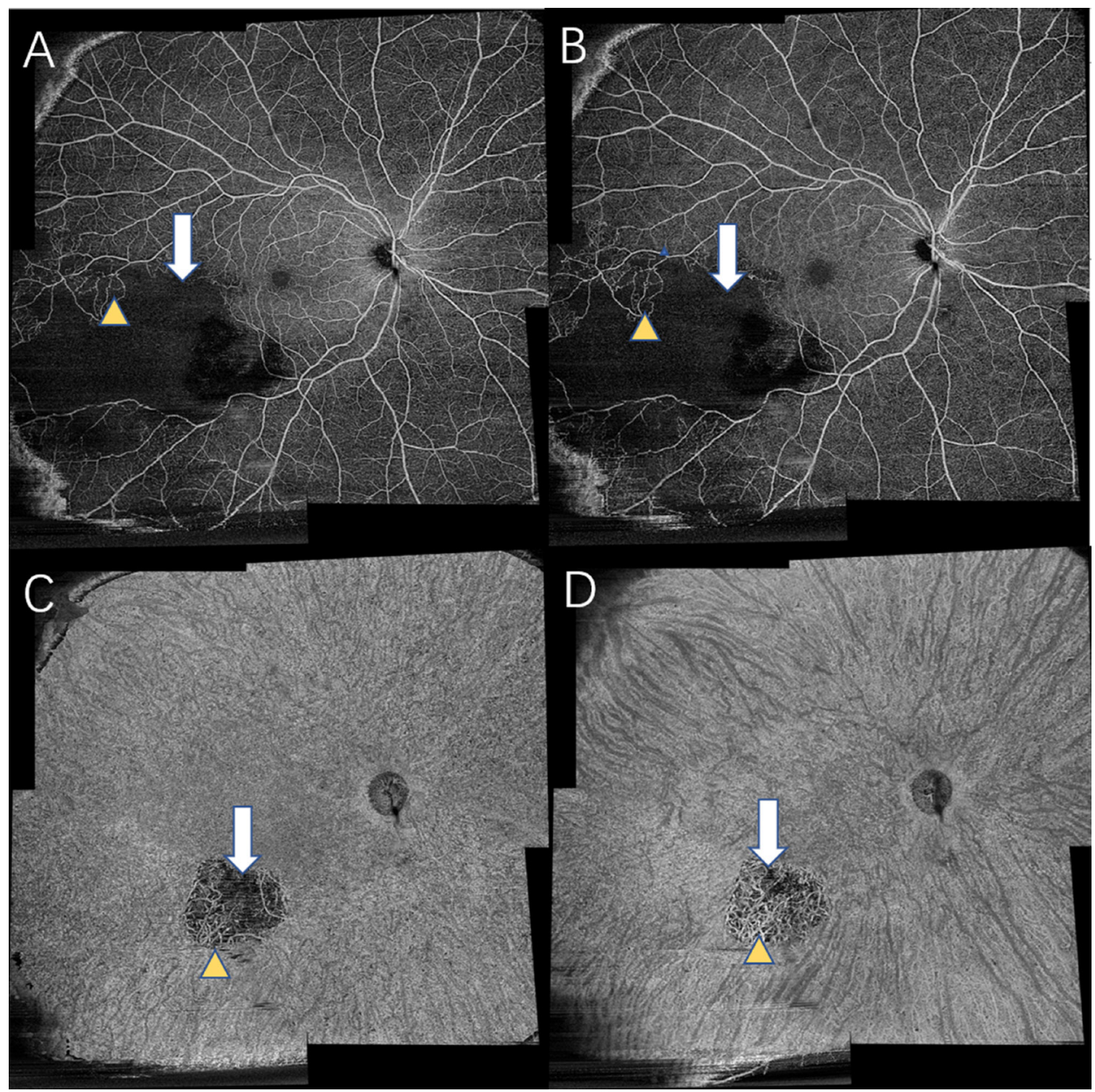

Figure 1 Non-perfusion (white arrow) and reduced perfusion (yellow triangle) in the montage scans. (A) Superficial capillary plexus, (B) deep capillary plexus, (C) choriocapillaris and (D) choroid. 


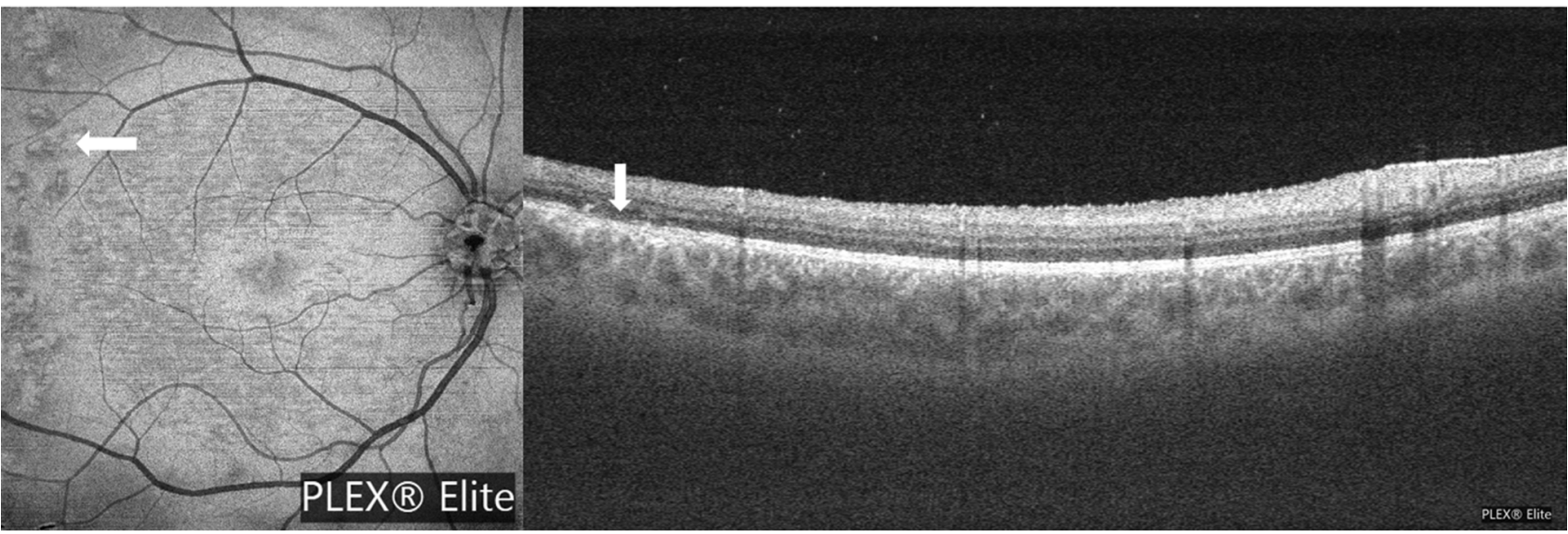

Figure 2 Disruption (=hyporeflectivity) on the ellipsoid zone of the en-face wide-field slabs. White arrows depict exemplary areas of ellipsoid zone disruption.

To analyse the individual vascular layers, the custom segmentation of the device was used. The superficial capillary plexus (SCP) extends from the internal limiting membrane (ILM) to the inner plexiform layer (IPL), and the DCP from the IPL (inner boundary) to the outer plexiform layer (outer boundary). The CC extends from $29 \mu \mathrm{m}$ beneath the retinal pigment epithelium(RPE) to $49 \mu \mathrm{m}$ beneath the RPE, and the choroidal layer has a thickness of $51 \mu \mathrm{m}$ and extends from $64 \mu \mathrm{m}$ to $115 \mu \mathrm{m}$ below Bruch's membrane. ${ }^{16}$

Qualitative analyses of wide-field OCTA images, en-face widefield slabs and thickness maps were independently conducted by two masked Bern Photographic Reading Center trained readers (MT and MRM), at different time-points and in different orders. In cases of discrepancies a consensus grading was performed. All images were evaluated on the instrument display screen in a standardised, dimmed environment. Wide-field montage OCTA images were examined for the presence of capillary non-perfusion and reduced capillary perfusion in SCP, DCP, CC and choroid. Capillary non-perfusion was defined as an area of total and profound capillary loss $\geq 1 / 4$ of the disc area ${ }^{9}$ (figure 1). Reduced capillary perfusion referred to smaller areas of reduced capillary density (figure 1). The individual structural OCT B-scans were evaluated for the presence of cystoid macualr oedema(CME), epiretinal membrane and disruption of the ellipsoid zone. The en-face wide-field slabs were individually examined for the presence of disruption of the ellipsoid zone (figure 2). The en-face wide-field retinal thickness maps (boundaries ILM and RPE; figure 3) were examined for the presence of perivascular thickening, macular thickening, peripapillary thickening, generalised thickening and retinal thinning (figure 4). The degree of retinal thickness was graded according to the colourcoded thickness maps. Cool colours (green and blue) represent decreased thickness, while warm colours (yellow, orange and red) represent increased thickness. ${ }^{17}$ Qualitative analysis of capillary network abnormalities was then compared with FA. Peripheral capillary leakage was compared with flow changes on the wide-field OCTA. The $12 \times 12$ OCTA scans centred on the optic nerve head were carefully reviewed for non-perfusion/hypoperfusion and correlated to optic nerve head leakage visible on FA. Furthermore, the perfusion abnormalities found on the central $12 \times 12$ scans were correlated to macular leakage. The structural OCT B-scans were used to identify epiretinal membrane and . $\mathrm{CME}$

\section{Statistical analysis}

Statistical analysis of the data was performed using SPSS V.23.0. Descriptive statistics (percentages, means and SD) were computed for demographic and clinical variables. Intergrader reproducibility is indicated by Cohen's kappa. Kappa values up to 0.2 indicate a slight agreement, while values between 0.21 and 0.40 are considered as fair, $0.41-0.60$ as moderate, $0.61-$ 0.80 as substantial and $0.81-1$ as almost perfect agreement, respectively. ${ }^{18}$ Phi and Cramer's $\mathrm{V}$ was used for qualitative data analysis. A minimal value of $\mathrm{p} \leq 0.05$ was considered statistically significant. P value correction for multiple testing was employed using false discovery rate.

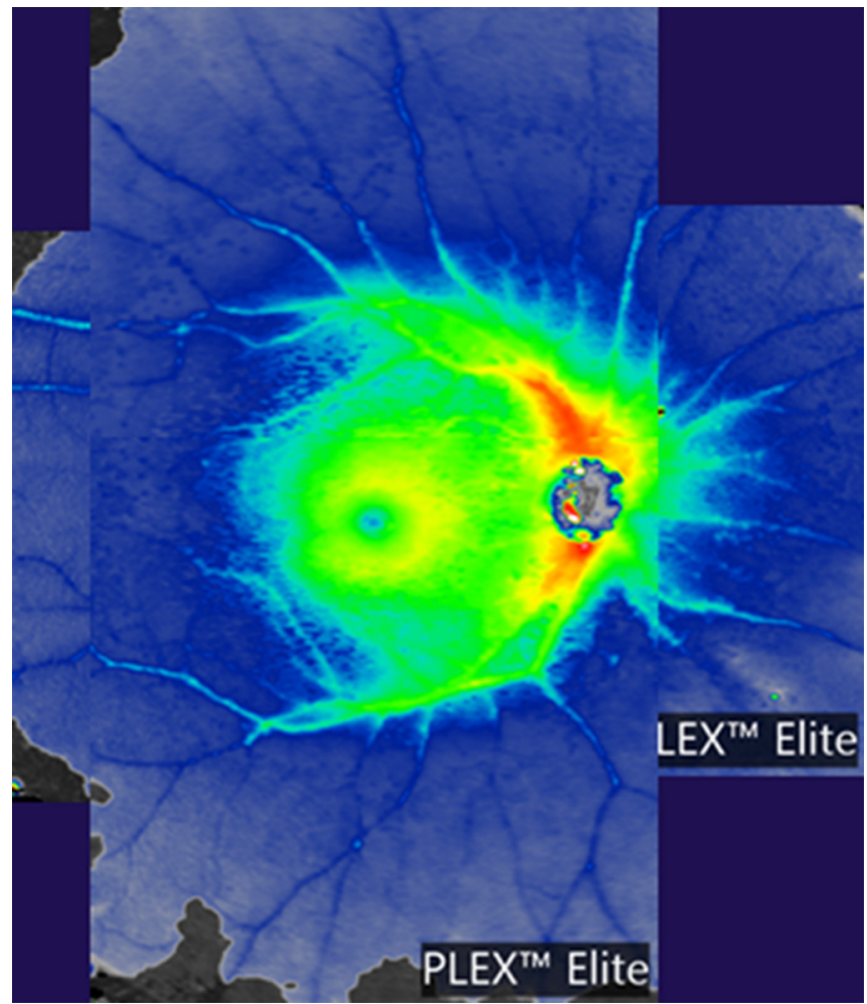

Figure 3 Example of colour-coded en-face wide-field retinal thickness map. 

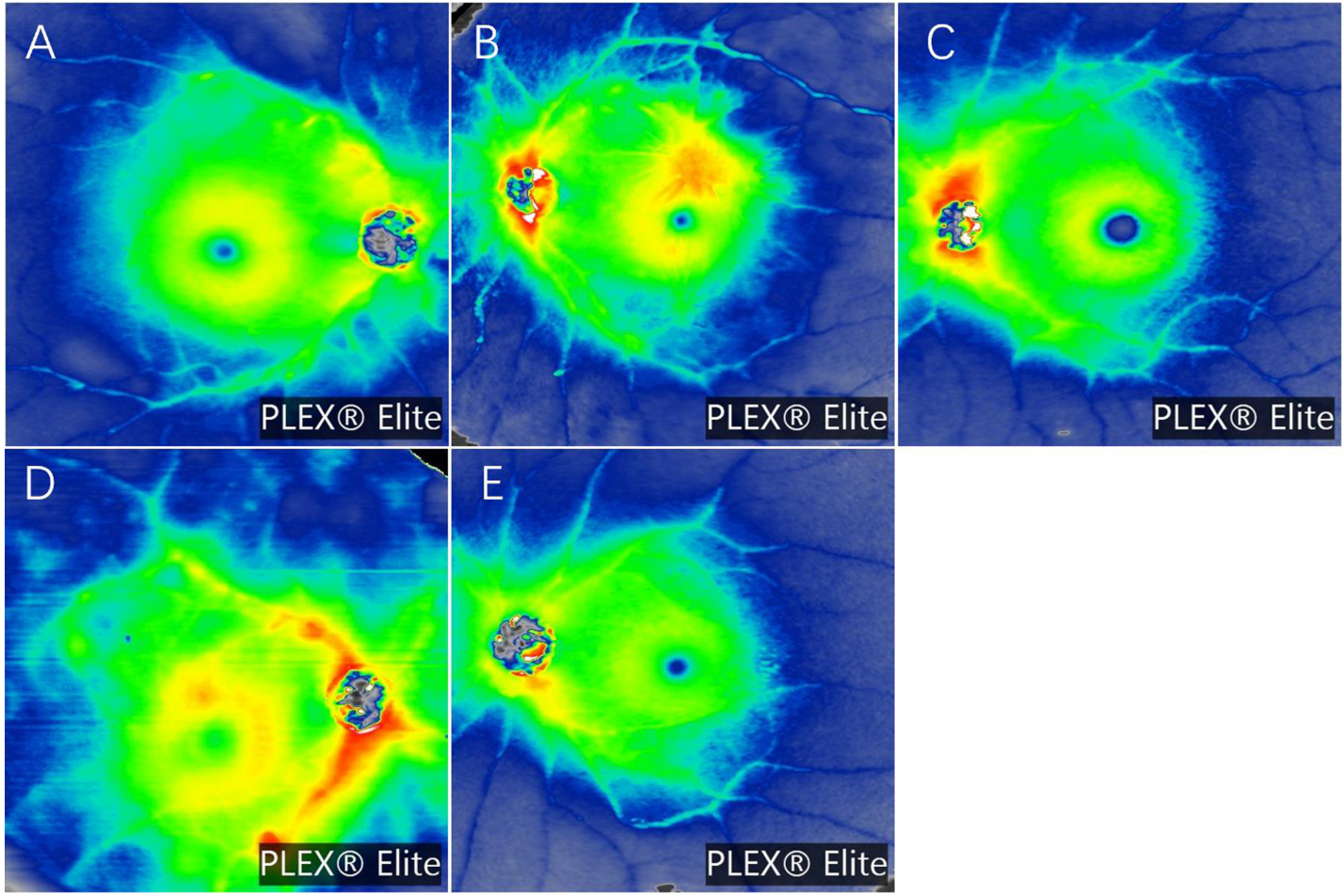

Figure 4 En-face wide-field thickness maps. (A) Normal, (B) macular thickening, (C) peripapillary thickening, (D) perivascular thickening and generalised thickening, and (

\section{RESULTS}

One hundred and sixty-four eyes from 88 patients were included in this study (mean age $45.5 \pm 18.7$ years, 54 women [61.4\%]). One hundred and twenty-four eyes $(75.6 \%)$ had IU and 40 eyes $(24.4 \%)$ had concomitant retinal vasculitis. The mean Snellen acuity was $0.9 \pm 0.5$ (range: $0.01-1.2$ ). Bilateral disease was found in $86 \%$. Thirty-six eyes $(22 \%)$ exhibited clinically active inflammation based on the SUN/NEI criteria. Forty-five patients $(51.1 \%)$ were on systemic conventional synthetic or biological disease-modifying antirheumatic drugs.

Eight eyes were retrospectively excluded from the analysis because of poor OCTA image quality. Thus, 156 (95.1\%) eyes, including 120 (76.9\%) with IU and 36 (23.1\%) with concomitant vasculitis, were evaluated. Out of these, 11 eyes have no FA images available. The intergrader agreement was substantial to almost perfect, ranging from 0.69 to 0.85 (Cohen's kappa). Online supplementary table 1 summarises the results of intergrader agreements for each individual evaluated parameter. Baseline clinical characteristics, FA and SS-OCT findings are summarised in table 1.

\section{En-face OCT wide-field findings}

The en-face wide-field thickness maps (figure 4) are summarised in table 2.

There was an association between peripheral capillary leakage on FA and the presence of perivascular, macular and generalised thickening on en-face wide-field thickness maps $(\mathrm{p}=0.007$, corrected [corr] $\mathrm{p}=0.04)$, but there was no significant correlation between clinically assessed disease activity and the findings on wide-field thickness map. Disruption of the ellipsoid zone was visible in 36 eyes (23.1\%) on the en-face wide-field slabs (figure 2), which was associated with the presence of CME ( $\mathrm{p} \leq 0.0001$, corr $\mathrm{p}=0.03)$ and ERM ( $\mathrm{p} \leq 0.0001$, corr $\mathrm{p}=0.03)$.

\section{Characteristics of eyes with IU with/without retinal vasculitis on wide-field OCTA}

Table 3 summarises the prevalence of capillary non-perfusion and reduced capillary perfusion on wide-field OCTA (figure 1) of eyes with IU with and without vasculitis in the individual customised retinal and choroidal slabs.

In general, capillary non-perfusion and reduced perfusion were more frequently observed in the choroid, CC and DCP than in the SCP (table 3). This high prevalence of CC and choroidal reduced perfusion seems noteworthy given that only five eyes

\begin{tabular}{|c|c|}
\hline Age (years) & $46 \pm 19$ \\
\hline \multicolumn{2}{|l|}{ Sex } \\
\hline Male & $36(41.4 \%)$ \\
\hline Female & $51(58.6 \%)$ \\
\hline \multicolumn{2}{|l|}{ Laterality } \\
\hline Unilateral & $5(5.7 \%)$ \\
\hline Bilateral & $82(94.3 \%)$ \\
\hline BCVA (decimal Snellen) & $0.9 \pm 0.5$ \\
\hline \multicolumn{2}{|l|}{ Baseline treatment } \\
\hline Disease-modifying antirheumatic drugs & $45(51.1 \%)$ \\
\hline \multicolumn{2}{|l|}{ Associated systemic disease/aetiology } \\
\hline Sarcoidosis & $20(12.2 \%)$ \\
\hline Latent tuberculosis & $8(4.9 \%)$ \\
\hline Behçet & $24(14.6 \%)$ \\
\hline Idiopathic & $112(71.8 \%)$ \\
\hline \multicolumn{2}{|l|}{ SS-OCT findings } \\
\hline Epiretinal membrane & $53(34 \%)$ \\
\hline Cystoid macular oedema & $28(18 \%)$ \\
\hline
\end{tabular}

BCVA, best-corrected visual acuity; SS-OCT, swept-source optical coherence tomography. 
Table 2 Findings on the en-face wide-field thickness maps

\begin{tabular}{ll}
\hline En-face wide-field thickness maps & Prevalence (\%) \\
\hline Perivascular thickening & $10(6.4)$ \\
\hline Macular thickening & $36(23.1)$ \\
Peripapillary thickening & $26(16.7)$ \\
Generalised thickening & $11(7.1)$ \\
Retinal thinning & $12(7.7)$ \\
\hline
\end{tabular}

(3.2\%) showed an area of obvious chorioretinal atrophy, which was always unrelated to underlying IU. While IU with vasculitis presented more frequently with non-perfusion and hypoperfusion in the DCP $(p=0.003$, corr $p=0.04$ and $p=0.05$, corr $\mathrm{p}=0.2$, respectively) and SCP $(\mathrm{p}=0.007$, corr $\mathrm{p}=0.04$ and $\mathrm{p}=0.005$, corr $\mathrm{p}=0.04$, respectively) than IU without vasculitis, there was no significant difference in terms of non-perfusion and hypoperfusion in the $\mathrm{CC}(\mathrm{p}=0.48$, corr $\mathrm{p}=0.7$ and $\mathrm{p}=0.79$, corr $\mathrm{p}=0.87$, respectively) or choroid between those two patient groups $(\mathrm{p}=0.1$, corr $\mathrm{p}=0.46$ and $\mathrm{p}=0.4$, corr $\mathrm{p}=0.7$, respectively) (table 3 ). There was no significant difference in capillary non-perfusion or reduced perfusion in the SCP $(p=0.5$, corr $\mathrm{p}=0.7$ and $\mathrm{p}=0.9$, corr $\mathrm{p}=0.93$, respectively) or DCP wide-field slabs $(\mathrm{p}=0.6$, corr $\mathrm{p}=0.74$ and $\mathrm{p}=0.2$, corr $\mathrm{p}=0.6$, respectively) between active and inactive eyes.

\section{Comparisons between OCTA and FA findings}

There were 47 cases with comparable FAs with a mean period between OCTA and FA of $10.7 \pm 14.7$ days. Details can be found in table 4A and B. Table 4A and B Comparison of wide-field FA findings and wide-field OCTA findings.

There was no association between peripheral capillary leakage on FA and non-perfusion or reduced peripheral perfusion on wide-field OCTA in the SCP $(\mathrm{p}=0.36$, corr $\mathrm{p}=0.7$ and $\mathrm{p}=0.88$, corr $p=0.93$, respectively), DCP $(p=0.94$, corr $p=0.94$ and $\mathrm{p}=0.7$, corr $\mathrm{p}=0.83$, respectively $), \mathrm{CC}(\mathrm{p}=0.5$, corr $\mathrm{p}=0.7$ and $\mathrm{p}=0.22$, corr $\mathrm{p}=0.6$, respectively) or choroid $(\mathrm{p}=0.47$, corr $\mathrm{p}=0.7$ and $\mathrm{p}=0.59$, corr $\mathrm{p}=0.74$, respectively). However, capillary dropout and ischaemia visible in $27.7 \%$ on FA was associated with capillary non-perfusion on wide-field OCTA in the SCP and DCP, respectively $(\mathrm{p}=0.019$, corr $\mathrm{p}=0.1$ and $\mathrm{p}=0.027$, corr $\mathrm{p}=0.12$, respectively), but missed statistical significance after $\mathrm{p}$ value correction. Considering FA as gold standard, the sensitivity and specificity in the detection of capillary dropout in the SCP on OCTA were 15\% and 97\%, respectively (false negative rate $=85 \%$, false positive rate $=3 \%$ ). The sensitivity and
Table 4A The wide-field FA findings in the subcohort compared with wide-field OCTA

\begin{tabular}{ll}
\hline Wide-field FA findings & Prevalence (\%) \\
\hline Peripheral capillary leakage & $36(76.6)$ \\
Peripheral capillary dropout & $13(27.7)$ \\
Hot disc & $15(31.9)$ \\
Macular leakage & $22(46.8)$ \\
\hline
\end{tabular}

FA, fluorescein angiography; OCTA, optical coherence tomography angiography.

specificity in the detection of capillary dropout in the DCP were 24\% and 94\%, respectively. Peripheral capillary non-perfusion or hypoperfusion of the CC and choroid was not associated with capillary dropout on FA (CC: $\mathrm{p}=0.39$, corr $\mathrm{p}=0.7$ and $\mathrm{p}=0.4$, corr $p=0.7$; choroid: $p=0.58$, corr $p=0.74$ and $p=0.31$, corr $\mathrm{p}=0.7$, respectively). Peripapillary capillary non-perfusion or hypoperfusion in the SCP $(\mathrm{p}=0.49$, corr $\mathrm{p}=0.7$ and $\mathrm{p}=0.7$, corr $\mathrm{p}=0.84)$, DCP $(\mathrm{p}=0.49$, corr $\mathrm{p}=0.7$ and $\mathrm{p}=0.13$, corr $\mathrm{p}=0.46)$, CC $(p=0.75$, corr $\mathrm{p}=0.86$ and $\mathrm{p}=0.49$, corr $\mathrm{p}=0.7)$ and choroid $(p=0.22$, corr $p=0.58$ and $p=0.5$, corr $p=0.7)$ was not associated with the presence of a hot disc. Also macular leakage on FA was not associated with non-perfusion or hypoperfusion on the central OCTA slabs.

\section{DISCUSSION}

We here present a prospective cross-sectional study using widefield SS-OCTA images to evaluate vascular changes in patients with IU with or without retinal vasculitis. Our paper evaluated vascular changes using wide-field OCTA in IU and retinal vasculitis as previous uveitis studies have focused on vascular changes on the central $3 \times 3 \mathrm{~mm}$ OCTA scans. ${ }^{19}{ }^{20}$ Our data show that montage wide-field OCTA seems to be a promising tool to detect microvascular alterations of the retina and choroid. Thus, flow void within $80^{\circ}-90^{\circ}$ can be directly seen and may not be indirectly evaluated using a central $3 \times 3$ or $6 \times 6$ scan. In contrast to FA, where the assessment of the capillary plexus is limited due to the trilaminar pattern of the network by leakage and by poor resolution of the DCP, ${ }^{320}$ OCTA can individually visualise the peripapillary plexus, the DCP, the CC and the choroid.

Qualitatively on the wide-field OCTA, the DCP was more frequently involved than the SCP. This is in line with the findings of previous studies ${ }^{12-14} 19$ on retinal diseases such as vein occlusion and diabetic retinopathy, in which more profound alterations have also been observed in the DCP than in the SCP. The reason for this may be that, unlike the superficial retinal

Table 3 Capillary non-perfusion and capillary reduced perfusion in SCP, DCP, CC and choroid

\begin{tabular}{|c|c|c|c|c|}
\hline & \multicolumn{2}{|c|}{ Intermediate uveitis without vasculitis } & \multicolumn{2}{|c|}{ Intermediate uveitis with concomitant retinal vasculitis } \\
\hline & Non-perfusion & Reduced perfusion & Non-perfusion & Reduced perfusion \\
\hline \multicolumn{5}{|c|}{ Montage wide-field scans } \\
\hline SCP & $3(2.5 \%)$ & $25(20.8 \%)$ & $5(13.9 \%)$ & $16(44.4 \%)$ \\
\hline DCP & $7(5.8 \%)$ & $37(30.8 \%)$ & $8(22.2 \%)$ & $17(47.2 \%)$ \\
\hline CC & $36(30 \%)$ & $57(47.5 \%)$ & $13(36.1 \%)$ & $18(50 \%)$ \\
\hline Choroid & $36(30 \%)$ & $57(47.5 \%)$ & $16(44.4 \%)$ & $20(55.6 \%)$ \\
\hline \multicolumn{5}{|c|}{ Peripapillary area } \\
\hline SCP & $1(0.8 \%)$ & $14(11.7 \%)$ & $0(0 \%)$ & $6(16.7 \%)$ \\
\hline DCP & $2(1.7 \%)$ & $13(10.8 \%)$ & $0(0 \%)$ & $7(19.4 \%)$ \\
\hline $\mathrm{CC}$ & $5(4.2 \%)$ & $12(10 \%)$ & $5(13.9 \%)$ & $12(33.3 \%)$ \\
\hline Choroid & $7(5.8 \%)$ & $16(13.3 \%)$ & $3(8.3 \%)$ & $12(33.3 \%)$ \\
\hline
\end{tabular}

CC, choriocapillaris; DCP, deep capillary plexus; SCP, superficial capillary plexus. 


\begin{tabular}{|c|c|c|}
\hline & Capillary non-perfusion & Capillary reduced perfusion \\
\hline \multicolumn{3}{|c|}{ Wide-field OCTA findings } \\
\hline SCP & $2(4.3 \%)$ & $12(25.5 \%)$ \\
\hline DCP & $4(8.5 \%)$ & $15(31.9 \%)$ \\
\hline $\mathrm{CC}$ & $15(31.9 \%)$ & $23(48.9 \%)$ \\
\hline Choroid & $16(34 \%)$ & $22(46.8 \%)$ \\
\hline \multicolumn{3}{|c|}{ Peripapillary area } \\
\hline SCP & $1(2.1 \%)$ & $8(17 \%)$ \\
\hline DCP & $1(2.1 \%)$ & $9(19.1 \%)$ \\
\hline $\mathrm{CC}$ & $4(8.5 \%)$ & $9(19.1 \%)$ \\
\hline Choroid & $3(6.4 \%)$ & $9(19.1 \%)$ \\
\hline
\end{tabular}

CC, choriocapillaris; DCP, deep capillary plexus; FA, fluorescein angiography; OCTA, optical coherence tomography angiography; SCP, superficial capillary plexus.

capillaries, the deep capillaries are not directly connected to arterioles, and are therefore more vulnerable to ischaemia. ${ }^{21} \mathrm{~A}$ previous paper by Spaide ${ }^{22}$ investigated the microvascular flow abnormalities associated with retinal vasculitis in areas of active vasculitis. Similar to our results, he found qualitatively larger areas of absent flow in the DCP compared with the SCP. ${ }^{22}$

Although wide-field OCTA seems a promising additional tool for the assessment of peripheral retinal ischaemia, it currently has limited potential to fully replace wide-field FA. Although the prevalence of reduced capillary perfusion found on the widefield OCTA in the SCP was similar to the prevalence of capillary non-perfusion on wide-field FA, the association of this finding between FA and OCTA did not remain statistically significant after $\mathrm{p}$ value correction. And although the specificity for the detection of capillary non-perfusion on OCTA was high, the sensitivity was rather low. However, our assessment was qualitative and a $12 \times 12$ scan pattern with a lower resolution of 24 $\mu \mathrm{m}$ was used. Previous studies could show that OCTA may be even more sensitive than FA in detecting perifoveal capillary abnormalities, such as hypoperfusion and non-perfusion on the ocular sarcoidosis eyes and retinal vasculitis, ${ }^{23} 24$ and a recent report illustrated that wide-field OCTA depicts the extensive capillary non-perfusion of the peripheral retina better than FA in some retinal vasculitis cases. ${ }^{25}$ Thus, with the progress of OCTA in terms of acquisition time and resolution, wide-field OCTA may soon become a sensitive and quantitative tool for the direct assessment of peripheral capillary dropout of the individual retinal and choroidal vascular layers.

Interestingly, although none of the OCTA data showed an association with peripheral capillary leakage on FA, there was a correlation between peripheral capillary leakage on FA and perivascular-peripapillary and macular thickening on en-face widefield thickness maps. In keeping, a previous study on patients with Birdshot chorioretinopathy has shown that perivascular thickening on thickness maps corresponds to vasculitis and may be used to follow phlebitis and disease activity in patients with respective disease. ${ }^{17}$ Our findings broaden the spectrum of evaluable diseases, which makes en-face thickness maps a promising, additional tool to follow patients with uveitis.

A surprisingly high percentage of patients had some CC and choroidal flow void. In some cases, alterations of the choroidal circulation were explainable by the presence of peripapillary chorioretinal atrophy or chorioretinal atrophy elsewhere; however, in the majority of eyes, decreased flow was independent of respective finding. Although several studies evaluated the choroidal involvement using OCTA in posterior uveitis, 42627 nothing is so far known about the choroidal involvement in patients with IU using OCTA. In a recent paper, Wintergerst and coauthors $^{28}$ quantitatively analysed the CC perfusion in central $3 \times 3 \mathrm{~mm}$ OCTA scans and also revealed that altered CC perfusion is frequently present in IU. The choroidal blood supply is still poorly understood. Anatomical postmortem studies describe the CC and choroid as highly anastomosed network, while in vivo studies indicate an end-arterial circulation. ${ }^{29}$ Whether IU and vasculitis without visible posterior involvement may cause indirect decrease in flow signal or a blockage phenomenon due to inflammation of the CC and choroid needs definitely further evaluations. Recent studies on healthy eyes quantified CC flow void and found an age-dependent decrease of CC in healthy subjects (oral presentation IRIS, Los Angeles, 2018; and OCT and Retina forum, Milan, 2018). ${ }^{30}$ It may be therefore possible that inflammation in the eye exaggerates the normal age-dependent decrease of CC and choroidal flow.

The inclusion of both eyes to increase the number of observations must be considered as potential limitation, as it may have biased our results. Overall, based on all the findings, we can conclude that OCTA is a valuable imaging modality to assess peripheral capillary non-perfusion in patients with IU with or without vasculitis and enables the evaluation of the perfusion status of the SCP, DCP, CC and choroid of about $80^{\circ}-90^{\circ}$.

Contributors MT: analysis of data, evaluation of data, writing the manuscript. TC: collecting data, critical review of the paper. MSZ: collecting data, evaluation of data, critical review of the paper. WH: statistical analysis, critical review of the paper. SW: financial support, collecting data, critical review of the paper. MRM: study design, analysis and evaluation of data, statistical analysis, critical review of the paper.

Funding The authors have not declared a specific grant for this research from any funding agency in the public, commercial or not-for-profit sectors.

Disclaimer MT: none. TC: consultant: AbbVie. WH: none. MRM: consultant: Novartis, Zeiss, GenSight Biologics and LumiThera; lecturer fees and travel support: Bayer. SW: consultant: Novartis, Zeiss, Heidelberg and Bayer. MSZ: consultant: Novartis, Heidelberg and Bayer; stocks: Novartis.

Competing interests None declared.

\section{Patient consent for publication Obtained.}

Ethics approval The study was approved by the University Clinic Bern IRB and followed the tenets of the Declaration of Helsinki.

Provenance and peer review Not commissioned; externally peer reviewed.

Open access This is an open access article distributed in accordance with the Creative Commons Attribution Non Commercial (CC BY-NC 4.0) license, which permits others to distribute, remix, adapt, build upon this work non-commercially, and license their derivative works on different terms, provided the original work is properly cited, appropriate credit is given, any changes made indicated, and the use is non-commercial. See: http://creativecommons.org/licenses/by-nc/4.0/.

\section{REFERENCES}

1 Bloch-Michel E, Nussenblatt RB. International Uveitis Study Group recommendations for the evaluation of intraocular inflammatory disease. Am J Ophthalmol 1987; 103:234-5

2 Khairallah M, Abroug N, Khochtali S, et al. Optical coherence tomography angiography in patients with behçet uveitis. Retina 2017;37:1678-91.

3 Spaide RF, Klancnik JM, Cooney MJ. Retinal Vascular Layers Imaged by Fluorescein Angiography and Optical Coherence Tomography Angiography. JAMA Ophthalmology 2015;133:45-50.

4 Pichi F, Sarraf D, Morara M, et al. Pearls and pitfalls of optical coherence tomography angiography in the multimodal evaluation of uveitis. J Ophthalmic Inflamm Infect 2017;7:20-32

5 Bouchenaki N, Cimino L, Auer C, et al. Assessment and classification of choroidal vasculitis in posterior uveitis using indocyanine green angiography. Klinische Monatsblätter für Augenheilkunde 2002;219:243-9.

6 Chalam KV, Sambhav K. Optical coherence tomography angiography in retinal diseases. J Ophthalmic Vis Res 2016;11:84-92.

7 Nesper PL, Roberts PK, Onishi AC, et al. Quantifying Microvascular Abnormalities With Increasing Severity of Diabetic Retinopathy Using Optical Coherence Tomography Angiography. Investigative Opthalmology \& Visual Science 2017;58:BI0307-15. 
8 Schaal KB, Munk MR, Wyssmueller I, et al. Vascular abnormalities in diabetic retinopathy assessed with swept-source optical coherence tomography angiography widefield imaging. Retina 2017;1:1-9.

9 Jia Y, Bailey ST, Wilson DJ, et al. Quantitative Optical Coherence Tomography Angiography of Choroidal Neovascularization in Age-Related Macular Degeneration. Ophthalmology 2014;121:1435-44.

10 Salz DA, de Carlo TE, Adhi M, et al. Select Features of Diabetic Retinopathy on SweptSource Optical Coherence Tomographic Angiography Compared With Fluorescein Angiography and Normal Eyes. JAMA Ophthalmology 2016;134:644-50.

11 Zhang Q, Lee CS, Chao J, et al. Wide-field optical coherence tomography based microangiography for retinal imaging. Scientific Reports 2016;6:22017-27.

12 Jabs DA, Nussenblatt RB, Rosenbaum JT, et al. Standardization of uveitis nomenclature for reporting clinical data. Results of the First International Workshop. Am J Ophthalmol 2005;140:509-16.

13 Nussenblatt RB, Palestine AG, Chan C-C, et al. Standardizatlon of Vitreal inflammatory Activity in Intermediate and Posterior Uveitis. Ophthalmology 1985;92:467-71.

14 Abu El-Asrar AM, Herbort CP, Tabbara KF. A clinical approach to the diagnosis of retinal vasculitis. International Ophthalmology 2010;30:149-73.

15 Abu El-Asrar AM, Herbort CP, Tabbara KF. Differential diagnosis of retinal vasculitis. Middle East Afr J Ophthalmol 2009;16:202-18.

16 Gao SS, Jia Y, Zhang M, et al. Optical Coherence Tomography Angiography. Investigative Opthalmology \& Visual Science 2016;57:0CT27-36.

17 Knickelbein JE, Tucker W, Kodati S, et al. Non-invasive method of monitoring retinal vasculitis in patients with birdshot chorioretinopathy using optical coherence tomography. Br J Ophthalmol 2018;102:815-20.

18 Landis JR, Koch GG. The measurement of observer agreement for categorical data. Biometrics 1977;33:159-74.

19 Kim AY, Rodger DC, Shahidzadeh A, et al. Quantifying Retinal Microvascular Changes in Uveitis Using Spectral-Domain Optical Coherence Tomography Angiography. American Journal of Ophthalmology 2016;171:101-12.
20 Pichi F, Sarraf D, Arepalli S, et al. The application of optical coherence tomography angiography in uveitis and inflammatory eye diseases. Progress in Retinal and Eye Research 2017;59:178-201

21 Park JJ, Soetikno BT, Fawzi AA. Characterization of the middle capillary plexus using optical coherence tomography angiography in healthy and diabetic eyes. Retina 2016;36:2039-50.

22 Spaide RF. Microvascular flow abnormalities associated with retinal vasculitis: A Potential of Mechanism of Retinal Injury. Retina 2017;37:1034-42.

23 Cerquaglia A, laccheri B, Fiore T, et al. New Insights On Ocular Sarcoidosis: An Optical Coherence Tomography Angiography Study. Ocular Immunology and Inflammation 2018;160:1-10.

24 Abucham-Neto JZ, Torricelli AAM, Lui ACF, et al. Comparison between optical coherence tomography angiography and fluorescein angiography findings in retinal vasculitis. Int J Retina Vitreous 2018;4:15.

25 Marchese A, Miserocchi E, Modorati G, et al. Widefield OCT Angiography of Idiopathic Retinal Vasculitis, Aneurysms, and Neuroretinitis. Ophthalmol Retina 2017;1:567-9.

26 Aggarwal K, Agarwal A, Mahajan S, et al. The Role of Optical Coherence Tomography Angiography in the Diagnosis and Management of Acute Vogt-Koyanagi-Harada Disease. Ocul Immunol Inflamm 2018;26:142-53.

27 de Carlo TE, Bonini Filho MA, Adhi M, et al. Retinal and choroidal vasculature in birdshot chorioretinopathy analyzed using spectral domain optical coherence tomography angiography. Retina 2015;35:2392-9.

28 Wintergerst MWM, Pfau M, Müller PL, et al. Optical Coherence Tomography Angiography in Intermediate Uveitis. Am J Ophthalmol 2018;194:35-45.

29 Lee JE, Ahn KS, Park KH, et al. Functional end-arterial circulation of the choroid assessed by using fat embolism and electric circuit simulation. Sci Rep 2017;7:2490.

30 Zhang Q, Zheng F, Motulsky EH, et al. A Novel Strategy for Quantifying Choriocapillaris Flow Voids Using Swept-Source OCT Angiography. Investigative Opthalmology \& Visual Science 2018;59:203-11. 